\title{
Corrigendum: Novel IncRNA IncFAM200B: Molecular Characteristics and Effects of Genetic Variants on Promoter Activity and Cattle Body Measurement Traits
}

\author{
Sihuan Zhang ${ }^{1}$, Zihong Kang ${ }^{1}$, Xiaomei Sun ${ }^{1,2}$, Xiukai Cao ${ }^{1}$, Chuanying Pan ${ }^{1}$, Ruihua Dang ${ }^{1}$, \\ Chuzhao Lei ${ }^{1}$, Hong Chen ${ }^{1}$ and Xianyong Lan ${ }^{1 *}$ \\ ${ }^{1}$ College of Animal Science and Technology, Northwest A\&F University, Yangling, China, ${ }^{2}$ College of Animal Science and \\ Technology, Yangzhou University, Yangzhou, China
}

Keywords: bovine, IncFAM200B, muscle development, promoter, body measurement traits

\section{A Corrigendum on}

Novel lncRNA IncFAM200B: Molecular Characteristics and Effects of Genetic Variants on Promoter Activity and Cattle Body Measurement Traits

by Zhang, S., Kang, Z., Sun, X., Cao, X., Pan, C., Dang, R., Lei, C., Chen, H., and Lan, X. (2019). Front. Genet. 10:00968. doi: 10.3389/fgene.2019.00968

Approved by:

Frontiers in Genetics Editorial Office,

Frontiers Media SA, Switzerland

*Correspondence:

Xianyong Lan lanxianyong79@126.com

Specialty section:

This article was submitted to

Livestock Genomics,

a section of the journal

Frontiers in Genetics

Received: 05 September 2021 Accepted: 28 September 2021

Published: 22 October 2021

Citation:

Zhang S, Kang Z, Sun X, Cao X, Pan C,

Dang $R$, Lei $C$, Chen $H$ and Lan $X$

(2021) Corrigendum: Novel IncRNA InCFAM200B: Molecular

Characteristics and Effects of Genetic Variants on Promoter Activity and

Cattle Body Measurement Traits.

Front. Genet. 12:770932.

doi: 10.3389/fgene.2021.770932
In the original article, there was an error. The analyses accession number in the DATA AVAILABILITY STATEMENT section was wrong.

A correction has been made to DATA AVAILABILITY STATEMENT:

The detailed information of SNP2 can be found in the European Variation Archive database. Project: PRJEB33081; Analysis Accession: ERZ3224744.

The authors apologize for this error and state that this does not change the scientific conclusions of the article in any way. The original article has been updated.

\section{DATA AVAILABILITY STATEMENT}

The detailed information of SNP2 can be found in the European Variation Archive database. Project: PRJEB33081; Analysis Accession: ERZ3224744.

Publisher's Note: All claims expressed in this article are solely those of the authors and do not necessarily represent those of their affiliated organizations, or those of the publisher, the editors and the reviewers. Any product that may be evaluated in this article, or claim that may be made by its manufacturer, is not guaranteed or endorsed by the publisher.

Copyright $\odot 2021$ Zhang, Kang, Sun, Cao, Pan, Dang, Lei, Chen and Lan. This is an open-access article distributed under the terms of the Creative Commons Attribution License (CC BY). The use, distribution or reproduction in other forums is permitted, provided the original author(s) and the copyright owner(s) are credited and that the original publication in this journal is cited, in accordance with accepted academic practice. No use, distribution or reproduction is permitted which does not comply with these terms. 